\title{
"Long-hissler" fine structure within auroral hiss: A review and synthesis
}

\author{
Howard F. Kim ${ }^{\mathrm{a}, *}$, James LaBelle ${ }^{\mathrm{a}}$, Maria Spasojević ${ }^{\mathrm{b}}$ \\ ${ }^{a}$ Dartmouth College Department of Physics, Hanover, NH 03755 \\ ${ }^{b}$ Stanford University, David Packard Bldg, 350 Serra Mall, Stanford, CA 94305-9515
}

\begin{abstract}
One of the most prominent fine-structures of auroral hiss is the "long-hissler", defined here as a dispersed feature embedded within broadband auroral hiss emissions in the frequency range $1-40 \mathrm{kHz}$ and lasting longer than 0.3 seconds. While theory is limited, there is evidence that hisslers can be used in remote sensing of density characteristics at altitudes of thousands of $\mathrm{km}$. By applying an automatic threshold algorithm to VLF data collected at South Pole 2230-0130 UT daily during June-August 2014, 22 hours of auroral hiss are identified on 49 of 93 days analyzed, for an occurrence rate of 9.7\% during the applicable MLT interval. From manual inspection of these intervals, 414 groups ("trains") of long hisslers are identified on 34 of the 49 days on which hiss occurred. Median lower (upper) frequency bounds of these features are $8(22) \mathrm{kHz}$, median frequency-time slope is $-10 \mathrm{kHz} / \mathrm{s}$, and median hissler repetition time within a train ("hissler period") is 1.2 seconds. Hissler period and frequency-time slope are inversely related. Data from previous studies are reviewed to provide a comprehensive description of the phenomenon. Contrary to some previous studies, subsequent long hissler features are found to commonly overlap in time, and no evidence is found for an inverse relationship between hissler train duration and geomagnetic activity.
\end{abstract}

Keywords: Hissler, Auroral Hiss, VLF, Dispersive Features, Ionosphere, Ground Observations, South Pole

\section{Introduction}

Studies of the ionosphere in the radio age, spanning roughly the past century, have revealed a host of very low frequency (VLF) phenomena with colorful names like whistlers, hiss, chorus, tweaks, etc. Prominent among these is auroral hiss, which was possibly observed as early as the 1930's (Burton and Boardman, 1933) and has since been the subject of intensive research summarized in several reviews (e.g. Sazhin et al., 1993; LaBelle and Treumann, 2002). Starting with the work of Makita (1979), auroral hiss has been categorized as either continuous or impulsive, the latter being relatively low bandwidth and lasting up to many hours, and the former lasting only minutes to tens of minutes at substorm onsets and extending to frequencies exceeding $1 \mathrm{MHz}$. The name "hiss" comes from the uniform sound produced when the VLF component of the waves are played through a loudspeaker, but on close inspection, auroral hiss exhibits various kinds of fine frequency structure, including banded features (e.g. Ye and LaBelle, 2008), irregular structures that have been interpreted as mode-converted auroral kilometric radiation (e.g. LaBelle and Anderson, 2011), and dispersive features called "hisslers" (Morozumi and Helliwell, 1966). Quasiperiodic emissions (QP) have a similar dispersive quality to hisslers but occur in a lower frequency range $0.5-5 \mathrm{kHz}$ (Smith et al., 1998; Engebretson et al., 2004). Hisslers have been subsequently classified by Siren (1974) into two types:

\footnotetext{
${ }^{*}$ Corresponding author

Email addresses: howardfk@gmail.com (Howard F. Kim), james.w. labelle@dartmouth. edu (James LaBelle), mariaspasojevic@stanford.edu (Maria Spasojević)
}

"fast" hisslers having time scales of milliseconds or tens of milliseconds (Siren, 1972, 1975), and "long" hisslers having longer time scales (Ungstrup and Carpenter, 1974).

Long hisslers are the focus of this review. Only a handful of papers have touched on these, theories of their generation mechanism remains unconfirmed. Long hisslers make their first appearance in Morozumi and Helliwell (1966), who describe them as periodic dispersive events embedded within hiss with frequency ranging from $8 \mathrm{kHz}$ to $4 \mathrm{kHz}$ over a duration of 4 seconds with activity lasting minutes. Carpenter et al. (1971) show an individual example of a falling-tone structure imbedded in hiss at $0-20 \mathrm{kHz}$, with $\sim 2$ second periodicity. Carpenter et al. (1971) cite the earlier technical report of Morozumi and Helliwell (1966) as well as a previous 1969 URSI presentation by Leif Owren. The first significant work on long hisslers was due to Ungstrup and Carpenter (1974) who show numerous examples and present statistical studies of characteristics using both ground-based and spacecraft-based data sets, the former from Byrd Station, Antarctica, and the latter from the Orbiting Geophysical Observatory (OGO) satellites 2 and 4. Much more recently, Sonwalkar and Harikumar (2000) show one example (their plate 1) and suggest a mechanism for long hisslers. A related PhD thesis (Harikumar, 2001) presents six additional examples observed at South Pole Station on five different days and presents details of the proposed mechanism supported by ray-tracing calculations.

Sonwalkar and Harikumar (2000) and Harikumar (2001) put forth a generation mechanism in which "long" hisslers arise from dispersion of oblique whistler waves generated through resonance with auroral electrons at altitudes of up to $10,000 \mathrm{~km}$ 
and subsequently scattered by meter-scale irregularities at altitudes of a few thousand kilometers. This mechanism implies that limits can be placed on electon densities and electron energy spectra at high altitudes based on "long" hissler dispersion and upper and lower frequency bounds. Further development of this theory could provide a useful tool for remotely sensing density profiles.

Section 2 details instrumentation, data collection, and analysis techniques regarding our recent observations. Section 3 reviews characteristics of long hisslers based on examples in both the recent study and in the previous literature. Section 4 summarizes the results.

\section{Method}

Experimental data reported below have been obtained from the Stanford University VLF wave receiving system at the Ammundsen-Scott South Pole Station (90 S geographic latitude; 74.3 invariant latitude). The wave sensor consists of a triangular, air-core, loop antenna with an area of $81 \mathrm{~m}^{2}$ located less than $1 \mathrm{~km}$ from the station. The receiving electronics bandlimits the data to $40 \mathrm{kHz}$ and converts the analog waveform to digital 16-bit words at $100 \mathrm{kHz}$ sample rate. The system has a long heritage, having been operated by Stanford using various receiver technologies for several decades. In austral winter 2014 , the system was operated such that waveforms were continuously digitized at $100 \mathrm{kHz}$ for three hours, 2230-0130 UT, each day. (For reference, midnight MLT is 0350 UT at South Pole Station, so this interval corresponds to the pre-midnight sector.) The resulting data, approximately 1.7 GB per day, were recorded onto digital versatile disc (DVD) eventually transported to Stanford University where they are archived. The data analyzed here are taken from May 31, 2014 to August 31, 2014. The experiment was largely successful, although on a few days data acquisition ended early.

Figure 1a shows an example $0-40 \mathrm{kHz}$ spectrogram recorded 0101-0112 UT on June 20, 2014. In this example Hamming windowed 4096-point Fast Fourier transforms have been applied to the waveform data, with $25 \%$ overlap between successive transforms, resulting in $24 \mathrm{~Hz}$ frequency resolution and 10 ms time resolution. The color scale represents approximately a $25-\mathrm{dB}$ range in relative power; calibrated results will be presented below. Figure 1a demonstrates that the experiment is sensitive to auroral hiss, which starts at 90 seconds and continues to the end of the record, with bandwidths ranging from a few $\mathrm{kHz}$ to nearly the full $40 \mathrm{kHz}$.

Figure $1 \mathrm{~b}$ shows a time series of a parameter used for automatic hiss detection: the sum of the logarithm of the wave power integrated over the frequency range $5-40 \mathrm{kHz}$, smoothed by application of a 2.04 second median filter. In contrast to a simple integrated power, this parameter, by summing log of power rather than power, effectively magnifies large wave intensities and produces greater contrast between times of auroral hiss and weak or absent hiss. Through trial and error, applying a threshold value of this parameter and comparing the resulting hiss identification with manual inspection of spectrograms, it was determined that a threshold of $-1.60 \times 10^{4} \mathrm{~dB}$ produces excellent reproducible automated detection of auroral hiss. This threshold value corresponds approximately to a wave amplitude of $1.1 \mathrm{pT}$; it is displayed with a red horizontal line in Figure 1b. Comparison of Figures $1 \mathrm{a}$ and $1 \mathrm{~b}$ shows the effectiveness of auroral hiss identification using this threshold.

The 11-minute time scale of Figure 1ab shows the regions of auroral hiss but has insufficient time resolution to resolve dispersed "hissler" features imbedded in the hiss. That task requires expanded spectrograms such as Figures 1c-d, which correspond to 30 seconds starting at 0111 UT, indicated by red break-out bars in the figure. The first half of this record shows examples of the dispersive, falling-tone features called hisslers. These usually last 1-3 seconds and occur in a "train" of up to a dozen or more similar features. In this example, the hisslers are distinct enough from each other, and from the background auroral hiss, to trace their leading edges from the highest to lowest detectable frequency. In Figure 1d, black dots represent such a trace for a representative hissler starting approximately $7 \mathrm{sec}-$ onds into the record. Individual black dots show single points at approximately the same frequency $(12 \mathrm{kHz})$ on the leading edges of each of the other hisslers comprising the hissler "train" in this example.

The experiment data set consisting of approximately 226 hours of data were analyzed in the following manner. First, the threshold criterion described above was applied to identify times of auroral hiss, resulting in a reduced data base of approximately 22 hours when hiss occurred (corresponding to an occurrence rate of $9.7 \%$ for auroral hiss within this favorable pre-midnight MLT sector). Next, the reduced data set was examined manually in 30-second intervals to identify hisslers. A custom graphical user interface (GUI) was created, which allows the user to tag times and frequencies of selected pixels in the spectrogram images of the structured hiss. The GUI also allows interactive variation of the color scale to highlight frequency or time-gradients in power spectral density. Hissler events were documented by tracing out the shapes of their leading edges with a series of mouse clicks recording selected frequency, power and time. For purposes of this study, a hissler is defined as an embedded structure with a duration greater than or equal to 0.3 seconds. As mentioned above, hisslers occur in quasi-periodic groups called "hissler trains." For purposes of this study, a "train" is defined as a set of consecutive hisslers for which no time interval between them exceeds 8 seconds. With these definitions, trains were identified, and for each train a representative hissler was traced completely from its highest to its lowest detectable frequency, as shown for the hissler starting at 7 seconds in Figure 1d. In addition, a single point at a selected common frequency was tagged for each hissler in the train. In the case of Figure 1d, $12 \mathrm{kHz}$ was selected corresponding to the approximate center frequency of most of the hisslers. (The selected frequency was different for each train depending on the data.)

the analysis method described above resulted in a database of 414 hissler trains distributed among the 22 hours of auroral hiss, implying an average occurrence rate of about one train per 3 minutes of hiss. Figure 2 shows spectrograms for seven 

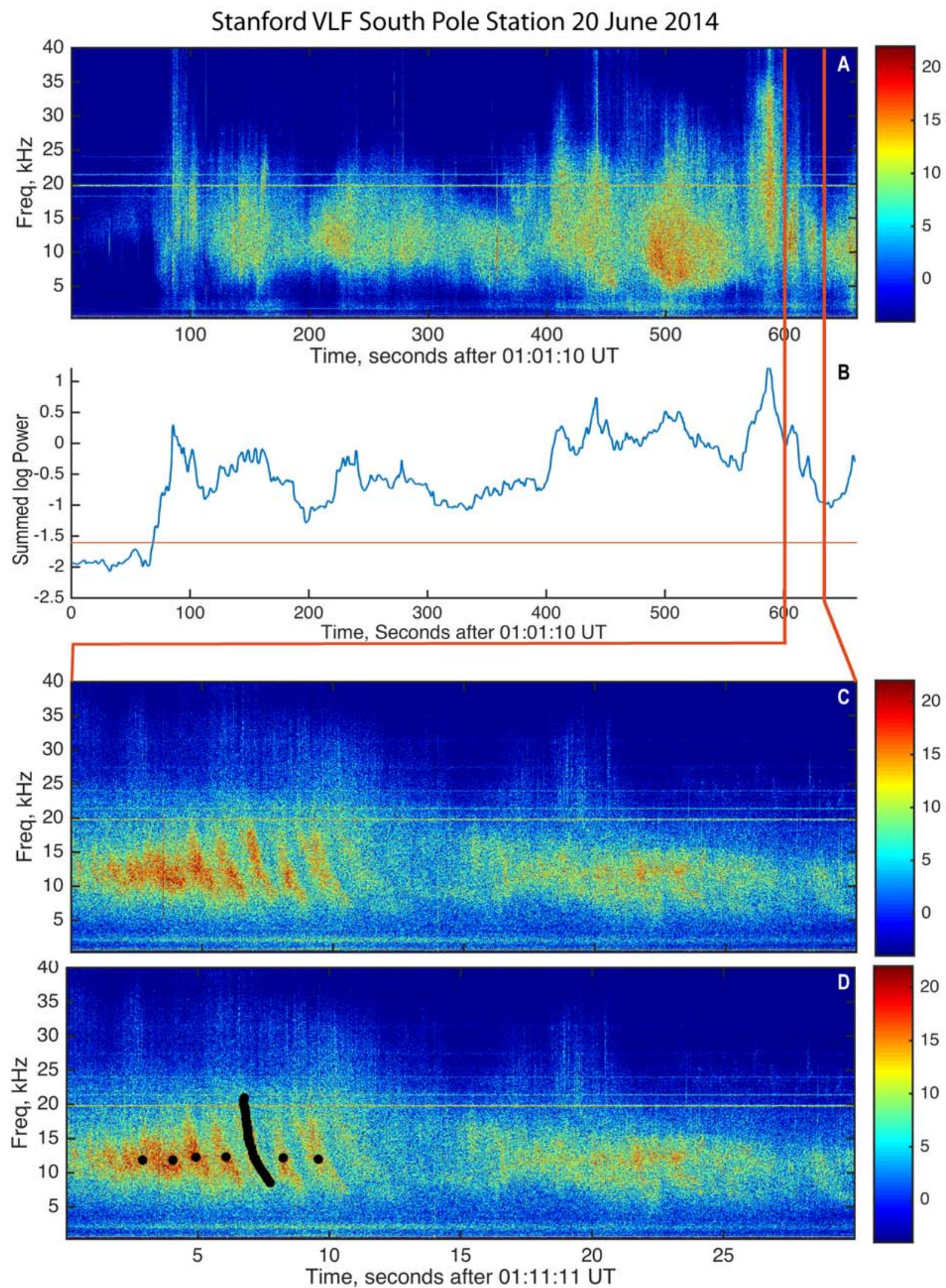

Figure 1: Panel A shows hiss on an 11 min window. Panel B shows the summed log power threshold used to identify hiss on the same 11 min scale. The red lines indicate where Panels C and D lie on the 11 min scale. Panels C and D show an example of one train event with seven hisslers. The two are identical, but the latter displays the manually collected data marked with black dots. The representative hisslers shape is marked completely, while the other six are marked just once to determine the periodicity. 
selected hissler trains, providing an impression of the range of structured features that meet the criteria described above. besides displaying a range of bandwidths, durations, and frequencytime slopes, as discussed below, figure 2 shows that in some cases hisslers can be irregularly-shaped or difficult to trace. for example, figures $2 \mathrm{a}$ and $2 \mathrm{~b}$, corresponding to 22 seconds after 0025 ut on july 7 and 0109 ut on july 20, respectively, show cases in which successive hisslers in the "train" differ in frequency-time slope or shape. also, the time intervals between hisslers in the train vary. figures $2 \mathrm{ab}$ and $2 \mathrm{c}$, corresponding to 22 seconds after 2307 ut on june 19, show examples in which the leading edges of hisslers are difficult to identify due to the intensity of the background unstructured hiss. despite these challenges, the most distinctive central hissler was selected and traced, and single points on all the other hisslers tagged, as accurately as possible; obviously, care must be taken in the interpretation of the data in these cases. a "quality factor" 1-3 was assigned to each hissler train, which could be used later in sorting the data. the hissler trains illustrated in figure $2 \mathrm{df}$ were assigned a low quality factor (3), whereas most of the other examples of figure 2, for which hissler shape and spacing are regular, and the leading edges of the hisslers are relatively easy to identify and tag, were assigned higher quality factors (2-1), detailed in the caption. Out of the 414 hissler trains, 337 were of quality factor 3, 64 were of quality factor 2, and 13 were of quality factor 1 .

\section{Observational Results}

During the 93 day interval (May 31-August 31, 2014), the experiment collected 226 hours of viable data. Applying automated hiss detection using the method described above resulted in identification of 22.17 hours of auroral hiss (approximately $9.7 \%$ of the time during these premidnight hours). Hiss was identified on 49 of the 93 days, with an average duration of 27.2 minutes and a median duration of 10.9 minutes. Manual inspection of high resolution spectrograms during these 22 hours of hiss, as described above, yielded 414 hissler trains on 34 of the 49 days on which hiss occurred. The average duration of these hissler trains was 17.8 seconds, and their occurrence rate is therefore $9.2 \%$ of the time when hiss was identified and $0.88 \%$ of the time when the instrument collected data during the premidnight hours.

Figure $3 \mathrm{a}$ shows $\mathrm{K}_{\mathrm{p}}$ index for the experimental interval. Because data straddles hours 21-24 and 00-03, averaged values were used. Figure $3 \mathrm{~b}$ shows minutes of auroral hiss (blue trace) and numbers of hissler trains (green bars) as a function of day of year during the experiment. Hissler occurrence approximately tracks hiss occurrence; for example, of the six days on which the most hissler events were recorded, four of these correspond to the four days on which the most minutes of hiss were identified. Figure $3 \mathrm{c}$ shows a scatter plot of all 414 hissler trains as a function of day of year and time of day. Within the short 3hour local time window sampled, there is no evidence of local time dependence of hissler occurrence. Often many successive hissler trains occur on the same day. As explained above, in this study 8 seconds was used as the time separation defining separate hissler train events. Had a longer interval been used, fewer but considerably longer hissler trains would have been identified, including potentially three or four with durations exceeding one hour, as further discussed below.

Ungstrup and Carpenter (1974) provide no quantitative occurrence rates for auroral hiss or hisslers based on ground-based observations, but state that hisslers are a "relatively common phenomenon...detectable at Byrd Station, Antarctica on nearly every austral winter night." Based on OGO-4 satellite observations, however, they report observing hiss on $100 \%$ of passes and hissler events on $\sim 6-37 \%$ of passes and $\sim 25-65 \%$ of days, in the range of dates from March 2 to June 12, 1968. The satellite data are difficult to compare with ground-level data, since hiss wave vectors must fall within a narrow transmission cone in order to penetrate the Earth-ionosphere boundary to be observed on the ground. However, the daily hissler train occurrence rate, 34/93 (37\%) in the recent South Pole experiment is in the range reported by Ungstrup and Carpenter (1974) for satellite events. The recent data also seem consistent with a "relatively common phenomenon," although occurrence at South Pole (74 invariant latitude) is considerably less than every austral winter night as Ungstrup and Carpenter (1974) report for Byrd Station $\left(\Lambda 70^{\circ}\right.$ invariant latitude). Other published observations of hisslers analyze small numbers of events providing no occurrence statistics for comparison.

Figure 4 shows histograms of the minimum and maximum frequencies measured for the selected traced hissler for each of 414 hissler trains of this study. The frequency distribution peaks at $8 \mathrm{kHz}$ for minimum and $22 \mathrm{kHz}$ for maximum, implying a typical bandwidth of $12.75 \mathrm{kHz}$. The minimum frequency can be as low as $1.8 \mathrm{kHz}$, and on rare occasion the maximum extends to at least $40 \mathrm{kHz}$, the highest frequency to which the receiving system is sensitive. There is a fairly large spread (15-30 $\mathrm{kHz}$ ) in the distribution of maximum frequencies. It is challenging to compare these observations to previous experiments for which instrument sensitivity often only extended to $15 \mathrm{kHz}$ or so. For example, Ungstrup and Carpenter (1974) claim a typical bandwidth of $\sim 5 \mathrm{kHz}$, while also commenting on insufficient information to define the upper bound of frequencies, stating "few examples have been found to extend above $15 \mathrm{kHz}$ ". They show several examples in which the frequency ranges from $2 \mathrm{kHz}$ to $10 \mathrm{kHz}$ and a single example with a maximum frequency exceeding the spectrogram's range of $10 \mathrm{kHz}$. The upper bound of spectrograms shown in their paper is $10-12.5 \mathrm{kHz}$, though the text implies that at least some of their data extended to higher frequencies. Nevertheless, it seems likely that the significantly lower bandwidth of hisslers reported by Ungstrup and Carpenter results at least partly from instrumental limitations. Most of the other previous experiments suffer from similar limitations. Carpenter et al. (1971) shows a single example ranging from 4-15 kHz (their Figure 5c). Sonwalkar and Harikumar (2000) show a single example ranging from $\sim 7 \mathrm{kHz}$ to $\sim 17 \mathrm{kHz}$, which is the highest lower frequency bound reported in the literature and closest to our results. Harikumar (2001) looks at both fast hisslers and long hisslers with typical lower frequency from 7 $9 \mathrm{khz}$ and and upper frequencies from $15-20 \mathrm{kHz}$, with bandwidths from $6-13 \mathrm{kHz}$. Siren $(1972,1974,1975)$ reports on 

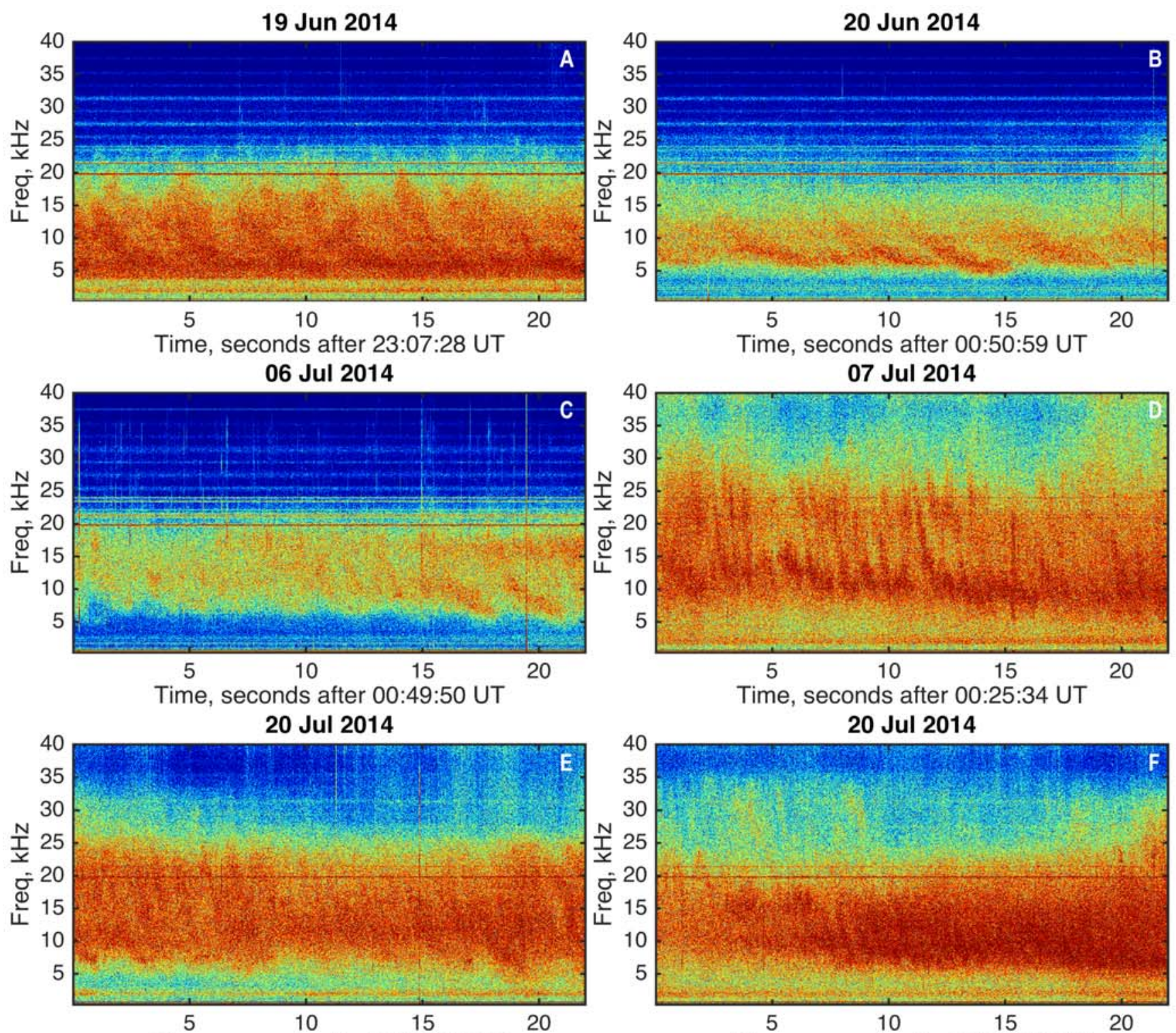

Time, seconds after 01:08:05 UT

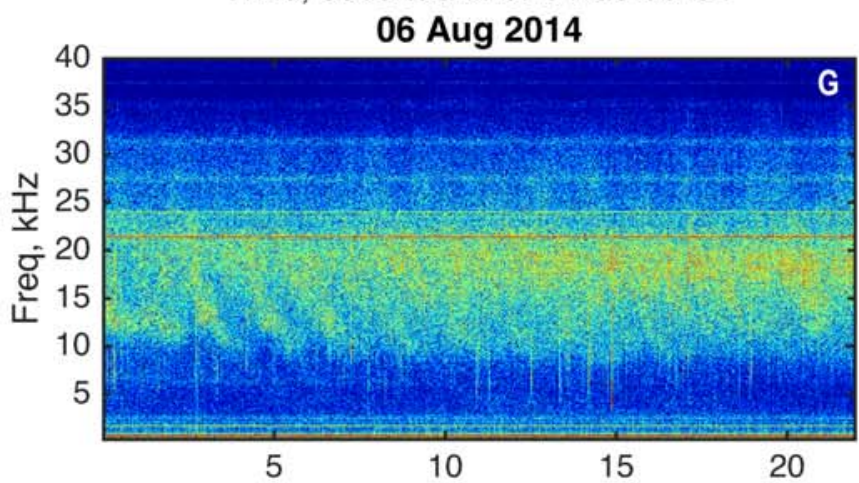

Time, seconds after 01:09:08 UT

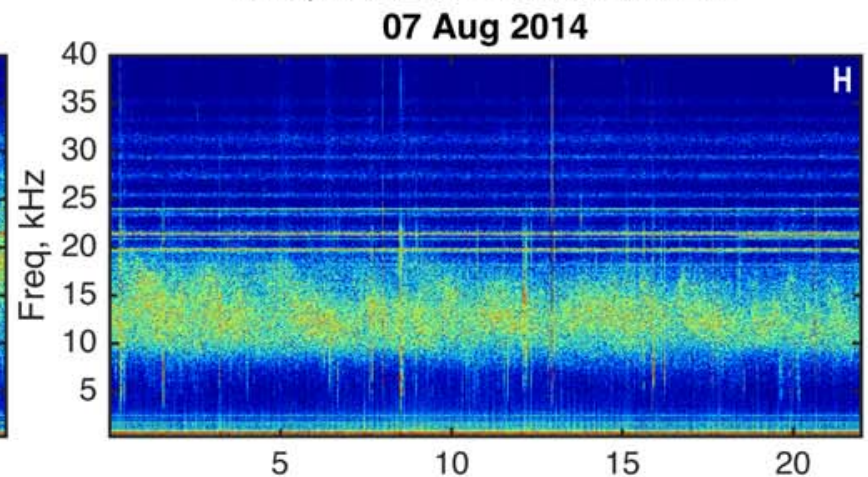

Time, seconds after 01:09:17 UT

Time, seconds after 00:58:42 UT

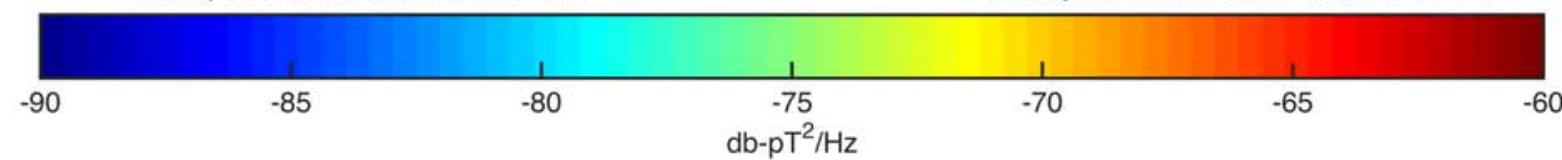

Figure 2: Eight representative spectrograms all scaled to the same time and frequency range of 22 seconds and $40 \mathrm{kHz}$, respectively. Panels D,E,F are marked for quality 3 while Panels C,H are quality 2, and A,B,G are quality 1 . 

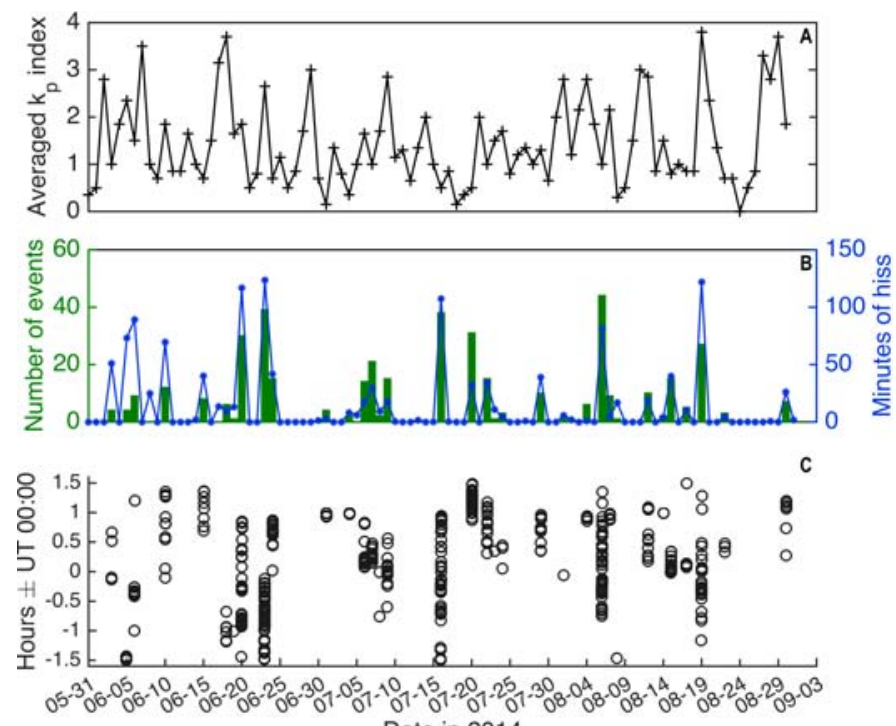

Date in 2014

Figure 3: Horizontal axes shows day of the year from 5-31 to 9-03. Panel A has averaged $K_{p}$ index for local time 12:00 am for each day. Panel B has a green bar graph that represent the number of hissler trains, while the blue line graph is the minutes of hiss. The green bar graph values correspond to the left vertical axis while the blue line graph values correspond to the right vertical axis. Panel $\mathrm{C}$ is the relative hours to local time 12:00 am for the start of each train event.

fast hisslers, a different but related phenomenon, with bandwidths $\sim 10 \mathrm{kHz}$, and minimum frequencies $\sim 2 \mathrm{kHz}$. Indeed, some previously reported minimum frequencies of hisslers are lower than the recent South Pole data base, around $2 \mathrm{kHz}$ versus mean of $8 \mathrm{kHz}$, though the frequency range and bandwidth from Harikumar (2001) is closer to the $8 \mathrm{kHz}$ to $22 \mathrm{kHz}$ range seen in the recent observations. Ungstrup and Carpenter (1974) show and remark on examples of frequency variability in sequential hissler events (for examples, their Figures 1, 2, and 4). In these cases the lower frequency bound of hisslers varies by a factor of 2-3, sometimes steadily increasing during the hissler train (see Figures 1 and 4 of Ungstrup and Carpenter (1974)) or rising and falling as in their Figure 2. This phenomenon was not quantified in the recent study. It occurred occasionally but was not typical. A majority of observed trains have hisslers with relatively uniform shapes and lower and upper frequency boundaries.

Figure 5a shows the distribution of the time separations between hissler events within a hissler train, also called the hissler period, and defined as the median time between hisslers in a each observed train. The average period is 1.9 seconds with a median of 1.7 seconds, and standard deviation of $0.85 \mathrm{sec}-$ onds. The distribution has maximum value just below $1.5 \mathrm{sec}-$ onds with $92.5 \%$ of the trains below 3 seconds and $7.5 \%$ in a tail extending to 8 seconds. The criterion used to define hissler trains, whereby gaps larger than 8 seconds imply a new train, precludes observation of periods longer than 8 seconds. At the other end of the range, instrumentation and the parameters for the FFT allow for a time resolution of 0.01 seconds, though typical hissler thicknesses of $0.2-0.5$ seconds imply a lower bound on the ability to distinguish individual hisslers in a train.

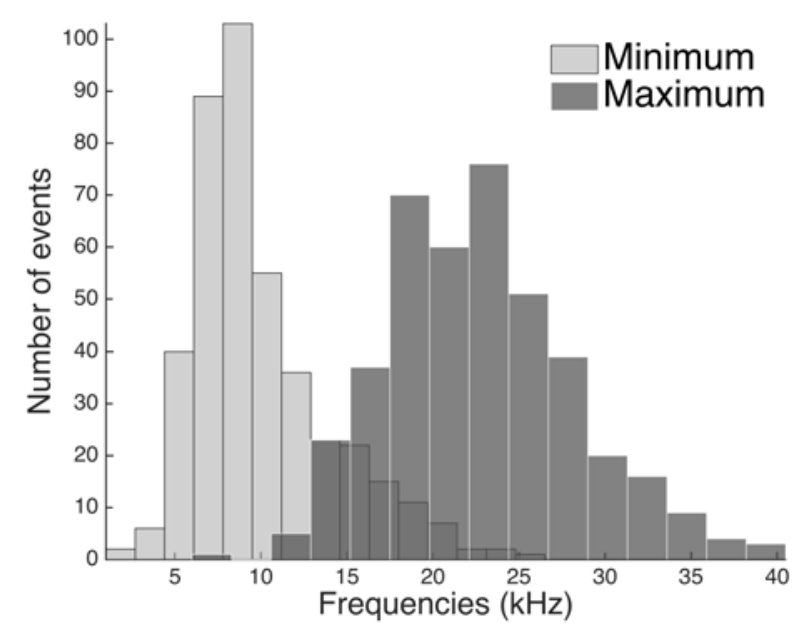

Figure 4: Two histograms are plotted with horizontal axes as frequency in $\mathrm{kHz}$ while the vertical access is number of hissler train events in each bin. Maximum frequency of the traced out shape is dark and the minimum frequency of the traced out shape is displayed as light.

The smallest period measured, 0.6 seconds, is not a limited by these considerations. For comparison, Ungstrup and Carpenter (1974) report that hissler periods range from 0.5 to 10 seconds with a typical value of 2-4 seconds. Sonwalkar and Harikumar (2000) and Carpenter et al. (1971) show examples with $\sim 2$ second time intervals between events. Harikumar (2001) examined six hissler trains on five days, with hissler periods ranging from 0.4-2 seconds; however, the criterion differed from that used by the other authors and possibly included fast hisslers on occasion. Examples of fast hisslers reported by Siren (1972, 1975) show considerably shorter periods of order 0.1 seconds. Accounting for this difference in criteria used in the Harikumar (2001) work, the previous experiments are consistent with the range of hissler periods shown in Figure 5a.

Figure $5 \mathrm{~b}$ shows the distribution of the number of hisslers per train. The distribution has its peak value just below 10 events, with a tail extending to 80 events. Combined with the data of Figure 5a, this distribution implies typical duration times for each train of 10-20 seconds, with the longest train lasting 105 seconds. For comparison, Sonwalkar and Harikumar (2000) show a single 30 seconds of a train that appears to continue beyond what is shown, and Carpenter et al. (1971) show an example of hissler train lasting 15 seconds. Harikumar (2001) shows 6 examples with duration $<54$ seconds.

In contrast, Ungstrup and Carpenter (1974) show one short example lasting less than a minute and several examples lasting on the order of 5 minutes in duration. Furthermore, they state that the "best-defined long-enduring ground observations ... last from tens of minutes to several hours" ( $p$ 5198). The discrepancy between those results and durations implied by Figure $5 b$ can probably be explained by differing criteria used in the two studies. In the current study, separations greater than 8 seconds implied a distinct hissler train. Ungstrup and Carpenter (1974) provide no clear definition for how they grouped events together into trains, but they likely used a more liberal criterion. By re- 

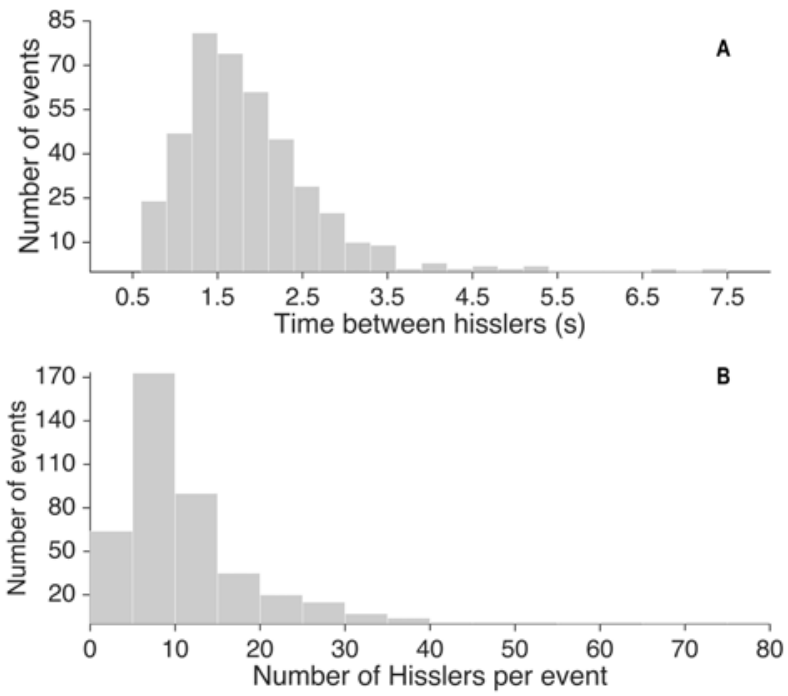

Figure 5: Vertical axis shows number of train events for each histogram. Panel A shows median time between hisslers in a train on the horizontal axis. Panel B shows a histogram of the number of hisslers per train.

defining hissler trains in the current study using 300 seconds rather than 8 seconds as the criterion implying a distinct train, the distribution changes considerably, with 20 of 108 trains lasting more than 7 minutes and several events lasting as long as 50 minutes. Somewhat more liberal criterion would imply several events exceeding one hour duration, as can be seen in Figure $3 c$.

Ungstrup and Carpenter (1974) suggest that longer train durations are associated with low $\mathrm{K}_{\mathrm{p}}$ values $(0-1)$. Figure $3 \mathrm{a}$ shows the $\mathrm{K}_{\mathrm{p}}$ during each two-hour interval in the current study, determined by averaging $\mathrm{K}_{\mathrm{p}}$ values for the two three-hour intervals straddled by the measurements. There is no clear correlation between low $\mathrm{K}_{\mathrm{p}}$ values and the occurrences of long hissler trains on June 23, July 16, August 05, and August 19. The correlation coefficients between duration of 108 hissler trains determined with the liberal 300 second criterion and $\mathrm{K}_{\mathrm{p}}$ yielded a correlation coefficient of just $\mathrm{R}=0.08$, suggesting no relationship between train duration and $\mathrm{K}_{\mathrm{p}}$ index. As a further test the 108 trains were split into two groups according to duration, the 54 shortest and 54 longest events. The Mann-Whitney test was applied to determine whether the distributions of $\mathrm{K}_{\mathrm{p}}$ values for these two sets are consistent with a single distribution. The test resulted in a p-value of 0.865 , which fails to reject the null hypothesis indicating the two groups are pulled from distributions with equal medians. These two tests reinforce the conclusion that no significant relationship exists between duration of the hissler trains and the $\mathrm{K}_{\mathrm{p}}$ index.

Figure 6 shows the distribution of the slopes of the selected hissler event for each of 414 observed hissler trains, defined as follows: for each selected hissler, sampled frequency-time points are fit to a second-order (three parameter) polynomial, and the slope is defined as the linear coefficient. The distribution of slopes ranges from -80 to $-1 \mathrm{kHz} / \mathrm{s}$ and peaks at approximately $-10 \mathrm{kHz} / \mathrm{s}$. Sonwalkar and Harikumar (2000) and

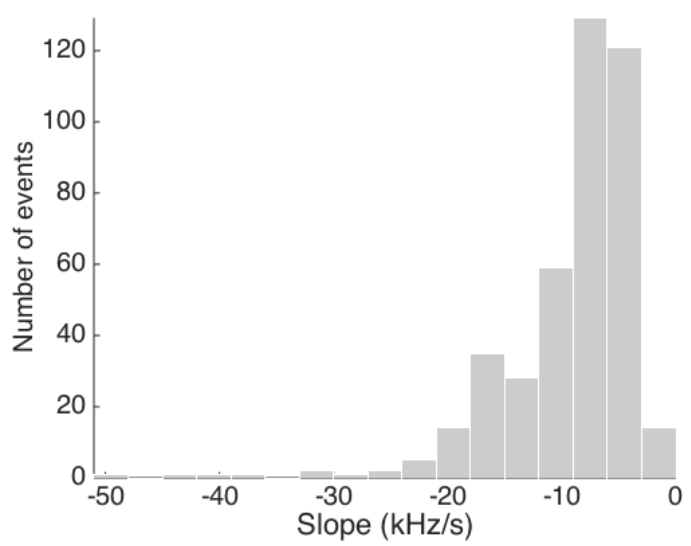

Figure 6: Histogram showing the slopes of the traced out example hisslers fitted to a second order polynomial fit.

Carpenter et al. (1971) show single examples in which the slope is about $-5 \mathrm{kHz} / \mathrm{s}$ and $-7 \mathrm{kHz} / \mathrm{s}$, respectively. Harikumar (2001) tabulates 22 events with slopes ranging from $-5 \mathrm{kHz} / \mathrm{s}$ to -24 $\mathrm{kHz} / \mathrm{s}$. As mentioned above, the Harikumar (2001) sample set is affected by a criterion that may include fast hissler events. (Fast hissler events, as shown by Siren $(1972,1975)$ have considerably steeper slopes of order $-100 \mathrm{kHz} / \mathrm{s}$.) From Figure 5 of Ungstrup and Carpenter (1974), it is possible to surmise a distribution of slopes. They calculated slopes using a linear relationship between the two starting and ending points of a hissler, and thus may have slightly different results compared to the second order fit using multiple points in this study; their slopes would range between -1.5 and $-10 \mathrm{kHz} / \mathrm{s}$. Hence, slope values in the previous literature, excepting those of Harikumar (2001) which may be contaminated by fast hisslers, lie generally on the shallow-slope end of the distribution determined in the current study. This discrepancy is probably related to the bias in maximum frequency, discussed above, related to the limited frequency range of many of the earlier observations. Hisslers show increasing slope at higher frequency, so observations biased toward lower frequency events will yield shallower slopes.

Correlations were sought between all measured hissler features, and only one significant correlation was found: that between hissler period and hissler slope. Ungstrup and Carpenter (1974) also reported a relationship between hissler period and slope. Figure 7b, a reproduction of Figure 5a of Ungstrup and Carpenter (1974), shows their result. Figure 7a shows the relationship between hissler slope and period determined from the more recent South Pole data. For this figure, the slope was determined from a linear rather than quadratic fit to the hissler shapes, in order to be consistent with how the fit was done in the work of Ungstrup and Carpenter (1974). In figure 7a, different color symbols represent different quality of the identified hisslers, as described in the methodology section above. In both panels, inverse slope magnitude is plotted versus hissler period. As discussed above, the Ungstrup and Carpenter (1974) data are biased toward steeper slopes due to inclusion of some "fast" hisslers. However, the two experiments unmistakably show 
the same trend: longer hissler periods correspond to shallower slopes (larger inverse slopes). In the case of Ungstrup and Carpenter (1974) the slope of these correlated data is $\sim 0.15 \mathrm{kHz}^{-1}$, while data from the current study yield a value closer to 0.045 $\mathrm{kHz}^{-1}$. However, if only the high quality (quality $>2$ ) hissler determinations are applied, then the slope of the correlated data in Figure $7 \mathrm{a}$ is $0.082 \mathrm{kHz}^{-1}$, closer to the value determined by Ungstrup and Carpenter (1974). Removing the outlier with the largest error bars in Ungstrup and Carpenter's data (figure 7b) gives correlated slope $0.13 \mathrm{kHz}^{-1}$ closer to agreement with the slope implied by the more recent South Pole data.

Fast hisslers reported by Siren (Siren, 1972, 1975) have a slope on the order of $100 \mathrm{kHz} / \mathrm{s}$; it is unclear whether these are simply cases of extreme values or a separate class of hisslers. This study focused exclusively on the "long hisslers"; we did not look at a scale that could reveal fast hissler phenomena at 0.1 seconds. Figure 5a shows a distribution of periods that cuts off at 0.6 seconds, and the shortest long hissler lifetime is 0.3 seconds. We might see the period distribution fill out the left tail, if adding fast hisslers to the distribution. The fast hisslers also appear to follow the trend in figure 7 showing inverse relationship between slope and period. Speculation might lead us to believe fast hisslers are extreme values of the same phenomena, however additional data and study on fast hisslers is needed.

Ungstrup and Carpenter (1974) claim that there is a nonoverlapping condition between hissler events in their study; that is, within a hissler train, each individual hissler event ends (at low frequency) before the next event starts (at high frequency). This characteristic can be seen in examples shown in their Figures 1, 2, and 4. The single event observed by Carpenter et al. (1971) does not contradict this non-overlapping hypothesis, but an event shown by (Sonwalkar and Harikumar, 2000, Plate 1c) does appear to contradict it. In the current study, a single selected hissler event was traced in each of 414 observed hissler trains, and the positions of all other hissler events in each train were also measured. Inferring that each hissler event in each train has the same shape and frequency extent as the selected one implies that 151 trains out of $414(36.47 \%)$ exhibit overlap between hissler events. Making fewer assumptions, one can compare the extent of the single selected hissler event in each train with the central location measured for the subsequent hissler event; this extremely conservative approach implies that 48 trains out of 414 (11.59\%) exhibit overlap between hisslers. The actual value probably lies between these two extremes, but is clearly not zero. As further evidence of that, Figures $2 b$ and $2 \mathrm{~g}$ show specific examples of hisslers observed on June 20, 2014, and August 6, 2014, in which overlap occurs. This combination of evidence eliminates the hypothesis that hisslers arise in such a way that successive events do not overlap with each other.

Following Makita (1979), and as reviewed by Sazhin et al. (1993), auroral hiss has generally been classified into two categories: impulsive hiss, which typically lasts for a few minutes at the time of substorm onset, and which has a large bandwidth of tens of $\mathrm{kHz}$ occasionally extending to above $1 \mathrm{MHz}$; and continuous hiss which lasts for much longer, tens of minutes to hours, and has small bandwidth, typically $\mathrm{kHz}$. Since the mech-

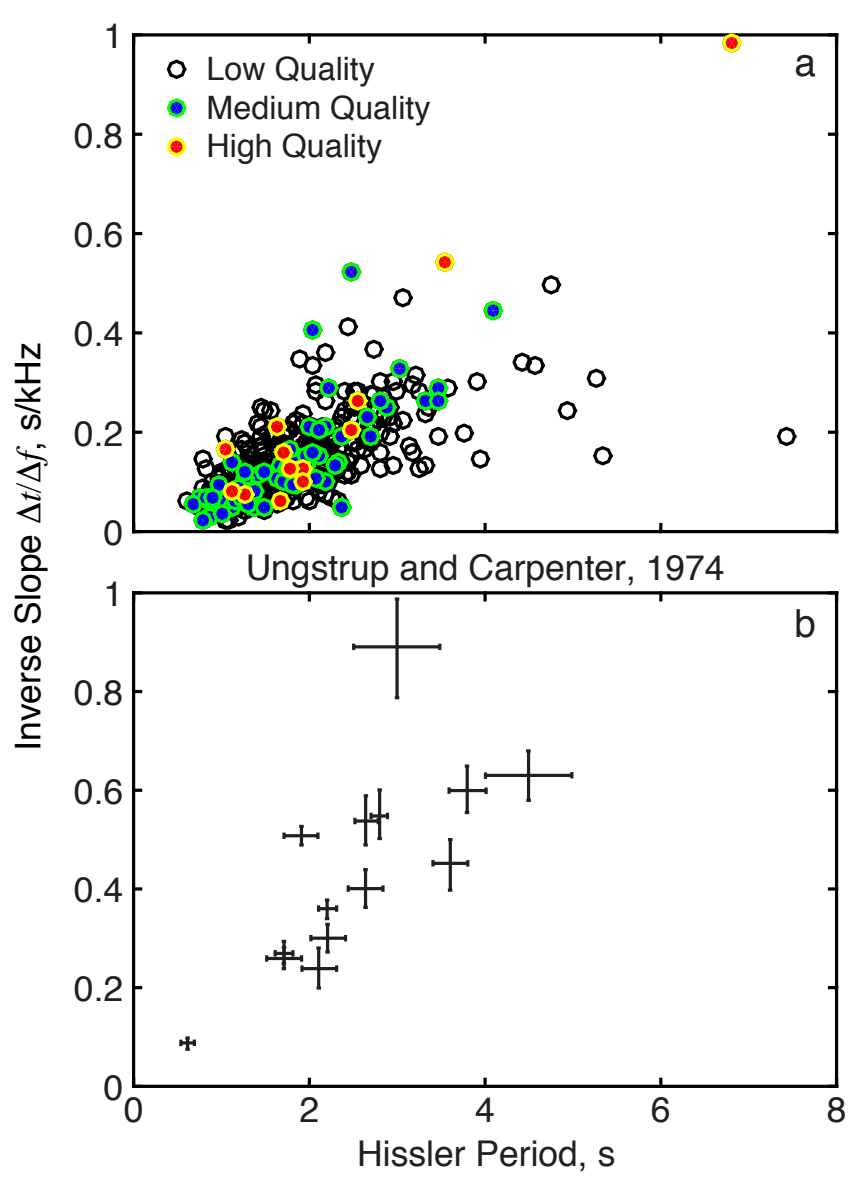

Figure 7: Shows the relationship between inverted slope and timing between hisslers. Panel a is our data while panel b is adapted from figure 5a of Ungstrup and Carpenter (1974) for comparison. Inverse slopes are magnatues.

anisms and source altitudes may differ between these, it is a significant question to determine which type is associated with hisslers. Of the 414 hissler trains identified in this study, 60 of them $(15 \%)$ occurred within 10 seconds of hiss that extended to the full $40-\mathrm{kHz}$ bandwidth of the instrument. Since continuous auroral hiss does not tend to exhibit such a large bandwidth, these appear associated with impulsive hiss, although in only one of these events did the hissler itself extend up to $40 \mathrm{kHz}$. On the other hand, four hissler trains of 414 hisslers $(0.01 \%)$ were associated with auroral hiss that had bandwidth less than $20 \mathrm{kHz}$ and lasted $30 \mathrm{~min}$. Since impulsive hiss does not normally last that long, these hissler trains seem likely to be associated with continuous hiss. In summary, those cases of hisslers which could be clearly associated with one type of hiss or the other suggest that the the vast majority of the hisslers occur in association with impulsive hiss, although they probably can occur in conjunction with continuous hiss as well. Proposed mechanisms for hisslers should therefore be applicable to both types of hiss. 


\section{Theory}

Relatively few papers touch on possible generation mechanisms for hisslers. Siren $(1972,1975)$ suggests that fast hisslers arise from dispersion during propagation of whistler modes with quasi-parallel wave vectors, implying very distant sources of order 25000 km. However, Sonwalkar and Harikumar (2000) criticize this theory on the grounds that quasi-parallel whistler modes cannot be generated via resonant processes with auroral electron beams that have energies at most tens of keV. Sonwalkar and Harikumar (2000) suggest, and Harikumar (2001) supports with ray-tracing calculations, a model whereby dispersion of oblique whistler modes, during propagation from source to the location of meter-scale irregularities which scatter the waves to near-parallel wave vectors, explains the hissler shape. They infer $3000-5000 \mathrm{~km}$ as the altitude of the scattering sites based on the lower frequency cutoff of the hisslers related to the lower hybrid frequency at the scattering location. The small amount of dispersion resulting from the parallel whistlers propagating below the scattering site is negligible compared to the group delay resulting from propagation of the oblique whistlers between source and scattering, which is calculated by Harikumar (2001) for a range of density profiles and source heights. There are enough free parameters in the model that the majority of hisslers with middle range of dispersions can be explained by various source height-density model combinations, making remote sensing of either quantity impossible without further information. However, relatively steep dispersions (short group delays) or shallow dispersions (long group delays) do allow definitive statements about the density profiles and source heights: in the former case, dispersions of 200-350 $\mathrm{ms}$, can only be explained by the lowest density magnetospheric model they use, with reference density of $0.1 \mathrm{~cm}^{-3}$ at $2 \mathrm{R}_{\mathrm{E}}$ altitude, with relatively low source heights of 5000-8000 km for the highest to lowest frequencies, respectively; in the latter case, dispersions exceeding 1 second can only be explained using the highest density model, with reference density of $100 \mathrm{~cm}^{-3}$ at $2 \mathrm{R}_{\mathrm{E}}$, and sources ranging from less than $8000 \mathrm{~km}$ for the lowest frequencies in the hissler to $>16000 \mathrm{~km}$ for the highest frequencies.

The recent South Pole observations can provide an estimate of what fraction of long hisslers fall into these two extreme dispersion categories. Out of 414 hisslers traced (one for each train), 75 of them $(18 \%$ ) have durations less than $350 \mathrm{~ms}$ for frequency spans of $5-10 \mathrm{kHz}$, and 211 of them $(51 \%)$ have durations that exceed 1 second for frequency ranges $4-12 \mathrm{kHz}$. This study gives an idea of the fraction of hisslers for which the density profiles and source heights can be inferred and take on the values described above, assuming the explanation of Sonwalkar and Harikumar (2000) is correct. Theory of the hisslers is clearly an underdeveloped field which could benefit from further investigations.

\section{Summary}

Auroral hiss includes complex spectra with different types of fine structure, including at least two different types of dis- persed features known as fast hisslers and long hisslers. This review focuses on long hisslers, defined as dispersed within the frequency range $1-40 \mathrm{kHz}$ and lasting longer than 0.3 seconds.

Current literature consists of only a handful of papers, some of which discuss single events. Past work has been augmented by a study of South Pole data from three months in austral winter, 2014. The recent data suggest that hissler events above 15 $\mathrm{kHz}$ are not as rare as implied in previous literature, probably due to instrumental restrictions in early studies. Hisslers as high as $40 \mathrm{kHz}$ are seen in the recent data set, with nearly half of the events starting above $20 \mathrm{kHz}$. The recent data also show a somewhat wider range of hissler periods, with a majority of events between 1 and 4 seconds and a tail extending to $8 \mathrm{sec}-$ onds.

Previous literature suggests a correlation between $K_{p}$ index and the occurrence of exceptionally long-duration trains which is not confirmed by recent data. Previous literature also suggests a "no overlap" condition between hisslers, in which individual hisslers terminate before the subsequent one starts. Recent data contradict this, showing evidence for overlapping events both in statistical studies and in case studies. The observed occasional overlapping implies that the time interval between long hisslers can be either smaller than or greater than the dispersion time of the hisslers at the location of observation. Any theory that posits no special dependence of the generation of hisslers on the dispersion time at a particular remote location would be consistent with this observation. In particular, this observation is consistent with the generation mechanism put forth by Sonwalkar and Harikumar (2000). Hisslers are seen during both impulsive and continuous hiss.

The theory of hisslers is not well developed, having been touched on in only one published paper in the open literature (Sonwalkar and Harikumar, 2000). Likewise, the relation between fast hisslers and long hisslers is uncertain. Even though auroral hiss is one of the longest-known ionospheric phenomena, any details of this phenomenon are not yet understood, including features with significant potential application to remote sensing of ionospheric plasma and processes.

\section{Acknowledgments}

Work at Dartmouth College was supported by NSF research grants PLR-1443338 and ANT-1141817. Work at Stanford University was supported by NSF award 1141791 . The $\mathrm{K}_{\mathrm{p}}$ index was provided by the Kyoto World Data Center. The authors thank the 2014 South Pole crew and science tech for overseeing successful operation of the VLF receiver. Thanks to Michelle Stephens for programing assistance and helpful discussions.

\section{References}

Burton, E., Boardman, E., Oct. 1933. Audio frequency atmospherics. AT\&T Tech. J. 12 (4), 498-516.

Carpenter, D., Fraser-Smith, A., Unwin, R., Hones, E., Heacock, R., 1971. Correlation between convection electric fields in the nightside magnetosphere and several wave and particle phenomena during two isolated substorms. J. Geophys. Res. 76 (31), 7778-7786. 
Engebretson, M., Posch, J., Halford, A., Shelburne, G., Smith, A., Spasojević, M., Inan, U., Arnoldy, R., 2004. Latitudinal and seasonal variations of quasiperiodic and periodic vlf emissions in the outer magnetosphere. Journal of Geophysical Research: Space Physics 109 (A5).

Harikumar, J., 2001. Investigation of auroral hiss observations on the ground: Application to remote sensing of auroral magnetosphere. Ph.D. thesis, University Alaska Fairbanks, https://sites.google.com/a/alaska.edu/waveslab/publications/theses.

LaBelle, J., Anderson, R. R., 2011. Ground-level detection of auroral kilometric radiation. Geophys. Res. Lett. 38 (4).

LaBelle, J., Treumann, R. A., 2002. Auroral radio emissions, 1. hisses, roars, and bursts. Space Sci. Rev. 101 (3-4), 295-440.

Makita, K., 1979. VLF-LF hiss emissions associated with aurora. Mem. of Natl. Inst. of Polar Res. Series A, Aeronomy 16, 1-126.

Morozumi, H., Helliwell, R., Dec. 1966. A correlation study of the diurnal variation of upper atmospheric phenomena in the southern auroral zone. Technical Report 2, Stanford University, http://vlf.stanford.edu/pubs/correlationstudy-diurnal-variation-upper-atmospheric-phenomena-southern-auroralzone.

Sazhin, S., Bullough, K., Hayakawa, M., 1993. Auroral hiss: A review. Planet. Space Sci. 41 (2), 153-166.

Siren, J. C., 1972. Dispersive auroral hiss. Nature 238 (86), 118-119.

Siren, J. C., Aug. 1974. Fast hisslers: A form of magnetosphereic radio emissions. Technical Report 3464-1, Stanford University, http://vlf.stanford.edu/pubs/fast-hisslers-form-magnetospheric-radioemissions-0.

Siren, J. C., 1975. Fast hisslers in substorms. J. Geophys. Res. 80 (1), 93-97.

Smith, A., Engebretson, M., Klatt, E., Inan, U., Arnoldy, R., Fukunishi, H., 1998. Periodic and quasiperiodic elf/vlf emissions observed by an array of antarctic stations. Journal of Geophysical Research 103, 23.

Sonwalkar, V. S., Harikumar, J., 2000. An explanation of ground observations of auroral hiss: Role of density depletions and meterscale irregularities. J. Geophys. Res. 105 (A8), 18867-18883.

Ungstrup, I., Carpenter, D., 1974. 'hisslers': Quasiperiodic (T 2 s) VLF noise forms at auroral latitudes. J. Geophys. Res. 79 (34), 5196-5201.

Ye, S., LaBelle, J., 2008. Ground based observations of low frequency auroral hiss fine structure. J. Geophys. Res. 113 (A1). 